\title{
Spinning fluid cosmologies in Einstein-Cartan theory
}

\author{
Larry L Smalley† and J P Krisch $\ddagger$ \\ $\dagger$ Department of Physics, University of Alabama in Huntsville, Huntsville, AL 35899, USA \\ $\ddagger$ Department of Physics, University of Michigan, Ann Arbor, MI 48109, USA
}

Received 6 June 1994

\begin{abstract}
We consider self-consistent spinning fluid cosmologies in both general relativity in Riemannian spacetimes and Einstein-Cartan theory in Riemann-Cartan spacetimes. First we extend slightly the cosmological calculation of Martin et al for general relativistic self-consistent spinning fuids. The existence of spin-squared terms in the field equations in the Einstein-Cartan theory shows, however, that an expanded class of meaningful cosmologies is possible. Under certain assumptions on the arbitrariness of the cosmological shear and expansion the results for the ad hoc Weyssenhoff spin fluid in a spherically symmetric spacetime can be reproduced.
\end{abstract}

PACS numbers: $0440,0450,9880$

\section{Introduction}

Self-consistent gravitational theories are generalizations of the Ray-Hilbert variational principle for perfect fiuids in general relativity [1] (GR) in the holonomic framework in which certain matter variables such as specific entropy and volume are also treated as thermodymical variables. The self-consistent formulation can easily be extended, for perfect fluids, to more complex spacetimes such as Riemann-Cartan (RC) spacetimes (which is the spacetime arena for the Einstein-Cartan theory (EC) [2]) or in general, a metricaffine spacetime (Poincare gauge theory) [3]. Some additional matter variables have been included such as spin density [4]; and for charged fluids in EC, the electromagnetic field [5-7]. Charged fluids have also been investigated and the theory extended to include the electromagnetic field as a thermodynamical variable [8].

Self-consistent spinning fluids have also been investigated in the holonomic framework in EC by de Ritis et al [9] in the anholonomic framework in EC by Obukhov and Korotky [10] and in GR by Obukhov and Piskareva [11]. Other variational principles are known but not considered here; for example, the Lagrange variational principle of Kopczynski [12] in EC which self-consistently yields both the matter currents and the equation of motion of the currents. On the other hand, the phenomenological approach does not rigorously yield the equations of motion since the currents are not defined in terms of the actual dynamical variables of the fluid (see, for example, discussions in [13]). However earlier cosmological models based upon the EC theory [14] using ad hoc prescriptions for torsion are known. See for example the discussion on a non-singular universe by Kopczyndski [15] using the Cartan relation between spin and torsion [16] or the discussion of early cosmological times [17], using the Weyssenhoff spin fluid model [18].

A general feature of the self-consistent spinning fluid theories in the holonomic frame is consistency conditions. They were first discussed by Amorim [5] for self-consistent, charged spinning fiuids in EC, with later corrections by Ray and Smalley [7]. Within this framework 
Gasperini has discussed spin-dominated inflation in EC theory [19]. More recently Martin et al [20] have discussed consistency conditions for a spinning fluid in GR [21]. However, Martin et al have indicated that within the cosmological context for a spinning fluid in GR [20], the consistency relation may limit useful solutions to the case of purely stationary spinning fluids. These consistency conditions seem to occur in all known solutions of selfconsistent theories in the holonomic framework [22-24] but do not seem to be a feature of the anholonomic framework because of a different formulation of the thermodynamics $[10,11]$. In principle these two distinct types of formulations should be equivalent. This seems to be the general importance of the consistency conditions.

We make the following observations.

(1) One must be careful in taking EC solutions to the GR limit. The limiting procedure in which one zeroes the spin contributions from the antisymmetric connections while keeping any explicit spin contributions from the stress energy content is incorrect. Given a set of self-consistent EC field equations, the correct limiting procedure will zero any spin squared terms in the EC equations, leaving all others as contributions to the self-consistent GR system. In $\mathrm{EC}$, the torsion field equation is given by [4]

$$
\hat{S}_{i j}^{k}=\frac{\kappa}{2} \rho s_{i j} u^{k}
$$

where the torsion is the antisymmetric part of the connection, $S_{i j}{ }^{k}=\Gamma_{[i j]}{ }^{k}$, the caret signifies the trace-free proper torsion, $\kappa=8 \pi G$, where $G$ is the gravitational constant, $\rho$ is the density, $s_{i j}$ is the spin density, and $u^{k}$ is the four velocity. Thus the limit $S_{i j}{ }^{k} \rightarrow 0$ gives GR. But by equation (1) in this limit, $s_{i j} \rightarrow 0$ as well, and one arrives at GR with a perfect fluid. A self-consistent spinning fluid in GR is not the simple limit of a self-consistent spinning fluid in an EC [20].

(2) The appropriate spacetime arena for spinning fuids is in EC in which a natural geometric object-the proper torsion-is directly related to the spin density as in equation (1). No such geometric object occurs in GR. We will see that the consequence of this observation is that no spin-squared terms, complementary to angular momentum squared terms can arise in the description of energy density of the spinning fluid. However spin-squared terms arise naturally for spinning fluids described in EC.

A self-consistent spinning fluid in $G R$ is a very different theory than a spinning fluid in EC†. Because of this we will show in this work that within EC, non-trivial cosmologies occur. In order to demonstrate this feature, we write the EC metric field equation in an effective-Einsteinian (EE) form by expanding the connection into Christoffel plus torsion parts and then replace the torsion using the torsion field equation (1). In this form the Bianchi identities then give the conservation laws.

In section 2, we revisit the GR calculation by Martin et al [20] and extend their results slightly as background to the EC case which is written in the EE form in section 3 . Application to spherically symmetric cosmologies for EC and results are discussed in section 4 , and we summarize our results in the last section.

\section{Spinning fluids in general relativity}

The self-consistent spinning fiuid formalism [20] gives the metric field equation

$$
G^{i j}=\kappa\left(T_{F}^{i j}+T_{s}^{i j}\right)
$$

$\dagger$ By ignoring the torsion field equation, an improper $G R$ limit can be obtained from the RC metric equation by putting the torsion to zero on the geometric 'fields' side and replacing the RC connection in the 'matter' side with the Christoffel connection. 
where $G^{i j}$ is the Einstein tensor (in GR), the perfect fluid energy-momentum tensor is

$$
T_{F}^{i j}=[\rho(1+\epsilon)+p] u^{i} u^{j}+p g^{i j}
$$

where $p$ is pressure, $\epsilon$ is energy density, and the spinning fluid energy-momentum tensor is

$$
T_{s}^{i j}=2 u^{(i} S^{j) k} \dot{u}_{k}+\nabla_{k}^{\prime !}\left[u^{(k} S^{i) k}\right]-\omega_{k}^{(i} S^{j) k}
$$

where the spin tensor $S^{i j}=\rho s^{i j}$, the overdot on the four velocity $\dot{u}_{k} \equiv u^{\ell} \nabla_{\ell}^{[\}} u_{k}$ represents the covariant derivative along fluid flow lines, $\nabla_{k}^{\prime \prime}$ represents the covariant derivative in GR with Christoffel connection $\left\{{ }_{i j}^{k}\right\}$, the spin angular velocity is

$$
\omega_{i j}=\dot{a}_{i}^{\mu} a_{\mu j}
$$

where $a^{\mu}{ }_{j}$ are a set of tetrads such that the metric is given by

$$
g_{i j}=\eta_{\mu \nu} a_{i}^{\mu} a_{j}
$$

$\eta_{\mu \nu}=(1,1,1,-1)$ is the Lorentz metric, and $a^{4 i} \equiv u^{i}$.

One finds that the spin density Fermi-Walker transports [25]

$$
\dot{s}_{i j}+2 u_{[i} s_{j] k} \dot{u}^{k}=0 .
$$

In the GR self-consistent formalism, the spin density is also treated as a thermodynamic variable so that the first law is given by [4]

$$
T \mathrm{~d} s=\mathrm{d} \epsilon+p \mathrm{~d}\left(\frac{1}{\rho}\right)-\frac{1}{2} \omega_{i j} \mathrm{~d} s^{i j}
$$

where $T$ is the temperature and $s$ is the specific entropy.

Dividing equation (8) by $\mathrm{d} x_{k}$ and contracting with $u^{k}$ gives the convective derivative of the thermodynamic law. In the self-consistent theories, entropy is conserved in the comoving frame, $\dot{s}=0$. We then have

$$
\dot{\epsilon}+p\left(\frac{1}{\rho}\right)^{\bullet}-\frac{1}{2} \omega_{i j} \dot{s}^{i j}=0 .
$$

The last term on the left-hand-side vanishes because of the definitions of the angular velocity and the spin density $s_{i j}=2 k_{0} a_{[i}^{1} a^{2}{ }_{j]}$ where $k_{0}$ is the constant magnitude of the spin vector in the comoving frame [26]. The expression given by equation (9) is also true in both GR and EC because of the Fermi-Walker transport of the spin density given by equation (7). Thus

$$
\dot{\epsilon}+p\left(\frac{1}{\rho}\right)^{\bullet}=0
$$

The conservation of particle number given by $\nabla_{i}^{\prime h}\left(\rho u^{i}\right)=0$ can be rewritten using the definition of the cosmological expansion parameter [27],

$$
\theta=\nabla_{i}^{0} u^{i}
$$


as

$$
\dot{\rho}+\rho \theta=0 .
$$

The consistency conditions are obtained from equation (2) using the Bianchi identity and contracting with $u_{i}$ so that

$$
0=u_{i} \nabla_{j}^{i}\left[T_{F}^{i j}+T_{s}^{i j}\right]
$$

Using equations (3), (10) and (12), the perfect fluid part is conserved automatically.

Before we give the consistency relation which follows from the spin part, we prove an identity. In EC, an identity for the antisymmetric components of the Ricci tensor [28] is

$$
R^{[i]}=G^{[i j]}=\nabla_{k} S^{i j k} .
$$

Taking the EC covariant derivative $\nabla_{j}$ of equation (14) and contracting with $u_{i}$ gives the EC equation

$$
u_{i} \nabla_{j} G^{[i j]}=\frac{\kappa}{2} \nabla_{j}\left[S^{\prime \prime} \dot{u}_{i}\right]
$$

But in Riemannian spacetime, $G^{[i j]} \equiv 0$, so that setting EC connection to Christoffel (see previous footnote) on the right-hand-side of equation (15) gives the GR equation

$$
\nabla_{j}^{\prime j}\left[S^{j i} \dot{u}_{i}\right]=0 .
$$

The spin part of equation (13) can be reduced to the equation

$$
0=u_{i} \nabla_{j}^{(j} T_{s}^{i j}=-\frac{1}{2} \nabla_{j}^{i l}\left(S^{j i} \dot{u}_{i}\right)-u_{i} \nabla_{j}^{(l)}\left[\omega_{\ell}^{(i} S^{j) \ell}\right] .
$$

Using equation (16) therefore gives the GR consistency relation

$$
u_{i} \nabla_{j}^{\prime \prime}\left[\omega_{\ell}^{(i} S^{j) \ell}\right]=0 .
$$

The cosmological shear parameter is given by [28]

$$
\sigma_{i j}=\nabla_{(j}^{(?} u_{i)}+\dot{u}_{(i} u_{j)}-\frac{1}{3} \theta\left(u_{i} u_{j}+g_{i j}\right)
$$

Expanding the covariant derivatives in equation (18), using the identity equation (16) plus the definitions for the cosmological parameters given by equations (11) and (19), the consistency relation becomes

$$
-\frac{2}{3} \omega_{i j} S^{i j} \theta+\omega_{k}^{i} S^{j k} \sigma_{i j}=0 .
$$

This is a slight generalization of the results of Martin et al in [20] where they leave unresolved the term given in the GR equation in equation (16).

In the next section, we investigate spinning fluid cosmologies in $\mathrm{EC}$. 


\section{Spinning fluids in Einstein-Cartan theory}

In EC the metric field equation now takes the form (assuming the torsion vector vanishes)

$$
G_{E C}^{(i j)}-2 \nabla_{k}\left[S^{k(i j)}\right]=\kappa\left[T_{P}^{i j}+T_{s}^{i j}\right]
$$

where the subscript EC refers to the Einstein tensor in EC, and where now $T_{s}^{i j}$ has the same 'form' as equation (4) except that covariant derivatives are in terms of the EC connection

$$
\Gamma_{i j}{ }^{k}=\left\{_{i j}^{k}\right\}+S_{i j}^{k}-S_{j}^{k}{ }_{i}+S_{i j}^{k} \text {. }
$$

We can rewrite equation (21) in a $\mathrm{EE}$ form by noting that the left-hand side becomes

$$
\begin{aligned}
G_{\mathrm{EC}}^{(i j)}-2 \nabla_{k} & {\left[S^{k(i j)}\right]=G^{i j}+2 S^{(i}{ }_{\ell n}^{j) \ell n}+S^{(i}{ }_{\ell n} S^{j) n \ell} } \\
& +S^{n k(i} S_{n k}{ }^{j)}-\frac{1}{2} g^{i j}\left[S^{\ell m n} S_{\ell m n}+2 S^{\ell m n} S_{\ell n m}\right] \\
= & G^{i j}-\kappa^{2}\left[\frac{1}{2} S_{\ell}^{i} S^{j \ell}+\frac{1}{4} S_{n \ell} S^{n \ell} u^{i} u^{j}-\frac{1}{8} g^{i j} S_{\ell m} S^{\ell m}\right]-\kappa \nabla_{k}^{(l}\left[S^{k(i} u^{j)}\right]
\end{aligned}
$$

where in the last two lines, we have used the torsion field equation (1). Similarly the right-hand-side of equation (21) becomes

$$
\begin{aligned}
\kappa\left[T_{P}^{i j}+T_{s}^{i j}\right]= & \kappa\left\{[\rho(1+\epsilon)+p] u^{i} u^{j}+p g^{i j}+2 u^{(i} S^{j) k} \dot{u}_{k}\right. \\
& \left.+\nabla_{k}^{(\}}\left[u^{(i} S^{j) k}\right]-\omega_{k}{ }^{(i} S^{j) k}-\kappa S_{k}^{i} S^{j k}-\frac{\kappa}{2} S_{k \ell} S^{k \ell} u^{i} u^{j}\right\}
\end{aligned}
$$

where we have used the identity for the spin angular velocity in an EC [29]

$$
\omega_{k C}^{k(i} S_{k}^{j)}=\omega^{k(i} S_{k}^{j)}-\frac{\kappa}{2} S_{k}^{i} S^{j k}
$$

The effective Einsteinian form now becomes

$$
\begin{aligned}
& G^{i j}=\kappa\left\{[\rho(1+\epsilon)+p] u^{i} u^{j}+p g^{i j}+2 u^{(i} S^{j) k} \dot{u}_{k}-\omega_{k}{ }^{(i} S^{j) k}\right\} \\
& -\kappa^{2}\left\{\frac{1}{2} S^{i}{ }_{k} S^{j k}+\frac{1}{4} S_{k \ell} S^{k \ell} u^{i} u^{j}+\frac{1}{8} S_{k \ell} S^{k \ell} g^{i j}\right\} .
\end{aligned}
$$

Using the GR results in the last section, the $\mathrm{EC}$ consistency relation is

$$
\begin{aligned}
0=u_{i} \nabla_{j}^{\prime\}} G^{i j}= & -\kappa u_{i} \nabla_{j}^{\prime l}\left[\omega_{k}{ }^{(i} S^{j) k}\right]-\kappa^{2} u_{i} \nabla_{j}^{\prime\}}\left[\frac{1}{2} S^{i k} S_{k}^{j}\right. \\
& \left.+\frac{1}{4} S_{k \ell} S^{k \ell} u^{i} u^{j}+\frac{1}{8} S_{k \ell} S^{k \ell} g^{i j}\right] \\
= & -\kappa u_{i} \nabla_{j}^{(\}}\left[\omega_{k}{ }^{(i} S^{j) k}+\frac{1}{2} \kappa S_{k}^{i} S^{j k}\right]
\end{aligned}
$$

where we have used the identity

$$
\left[S_{j k} S^{j k}\right]^{\bullet}=-2 \theta S_{j k} S^{j k}
$$

The last line in equation (27) can be rewritten in EC form [7] as

$$
u_{i} \nabla_{j}\left[\omega_{k}{ }^{(i} S^{j) k}\right]=0
$$

Using equation (19), we can put equation (27) in the final form

$$
\left[\frac{\kappa}{3} \omega_{j k} S^{j k}-\frac{\kappa^{2}}{6} S_{j k} S^{j k}\right] \theta+\left[\kappa \omega_{k}{ }^{(i} S^{j) k}-\frac{1}{2} \kappa^{2} S_{k}{ }^{i} S^{j k}\right] \sigma_{i j}=0
$$

which demonstrates that non-trivial cosmologies with both expansion and shear are possible in $\mathrm{EC}$. In the next section, by way of example, we investigate this particular consequence. 


\section{Einstein-Cartan cosmologies}

The consistency relation in EC equation (30) indicates that there are at least three distinct cosmologies possible. It is interesting that there is at most only a weak condition on the vorticity depending on both arbitrary expansion and shear; otherwise the vorticity is arbitrary. In this case, arbitrary expansion and shear lead to the conditions

$$
\frac{2}{3} \omega_{j k} S^{j k}-\frac{\kappa}{3} S_{j k} S^{j k}=0
$$

and

$$
\omega_{k}^{(i} S^{j) k}-\frac{\kappa}{2} S_{k}^{i} S^{j k}=0
$$

respectively. Note that the trace of equation (32) contains equation (31).

Consider the example of a spherically symmetric cosmology in $\mathrm{EC}$ spactime [30]. In this case, equation (21) can be written using equations (23) and (24):

$$
G^{i j}=\kappa\left[T_{F}^{i j}+T_{s}^{i j}+\tau^{i j}\right] \equiv \kappa T_{R}^{i j}
$$

where $T_{R}^{i j}$ is the effective spherically symmetric energy-momentum tensor with

$T_{F}^{i j}=[\rho(1+\epsilon)+p] u^{i} u^{j}+p g^{i j}$

$T_{s}^{i j}=-\frac{1}{3}\left[4 \Omega_{\ell k} S^{\ell k}+5 \kappa S^{2}-\omega_{\ell k} S^{\ell k}\right] u^{i} u^{j}-\frac{1}{3}\left[\Omega_{\ell k} S^{\ell k}+2 \kappa S^{2}-\omega_{\ell k} S^{\ell k}\right] g^{i j}$

$\tau^{i j}=\left[\frac{5}{6} \kappa S^{2}\right] u^{i} u^{j}+\left[\frac{1}{12} \kappa S^{2}\right] g^{i j}$

where the GR vorticity is defined as [27]

$$
\Omega_{i j}=-\nabla_{[j}^{[1} u_{i]}-\dot{u}_{[i} u_{j]}
$$

and $-\tau^{i j}$ is the spin-squared part of $G_{E C}^{(i j)}$ given in equation (23), and $S_{i j} S^{i j} \equiv 2 S^{2}$. The form of the average stress-energy tensor depends on assumptions about the fields and the various cosmological models that one can have in a spherically symmetric EC. If the consistency conditions are included in the analysis of the average value of equation (33), we can make definitive predictions. The average energy momentum tensor will depend on the particular assumptions about the type of cosmology being investigated. In taking the average of a spherically symmetric isotropic system of randomly oriented spins, the average of the spin itself is assumed to vanish, $\left\langle S^{i j}\right\rangle=0$, but for the spin-squared terms, $\left\langle S_{i j} S^{i j}\right\rangle \neq 0$. Since $S_{i j} S^{i j}=2 S^{2}$, where $S$ is the spin four-vector, then we set $\left\langle S^{2}\right\rangle \equiv S^{2}$ for convenience. For the case of a cosmology with arbitrary shear and expansion developed above, then the definition of the GR vorticity equation (37), defined in terms of the four-velocity, and the spin density, described after equation (9), shows that there is no correlation between the randomly oriented spins and the congruences associated with the four-velocity. We therefore assume that the average of the vorticity-spin term vanishes

$$
\left\langle\Omega_{j k} S^{j k}\right\rangle=0
$$

but on the other hand, equation (32) gives a non zero average for the angular velocity

$$
\left\langle\omega_{j k} S^{j k}\right\rangle=\kappa S^{2} .
$$


Using these results, the average of the energy momentum tensor equation (33) becomes

$$
\left\langle T_{R}^{i j}\right\rangle_{I}=\left[\rho(1+\epsilon+p / \rho)-\frac{1}{2} \kappa S^{2}\right] u^{i} u^{j}+\left[p-\frac{1}{4} \kappa S^{2}\right] g^{i j}
$$

where the subscript $I$ refers to the improved energy momentum tensor of the self-consistent spinning fluid in EC. This is identical to the results of Hehl et al [31] for the earlier EinsteinCartan theory for a Wessenhoff spinning fluid. In addition, the cases of no expansion but arbitrary shear, or vice versa, will give the same results. Note that equation (39) is equivalent to the condition that the average value of the $\mathrm{EC}$ angular velocity spin term vanishes.

Other cases are possible. For example, the case of no expansion or shear, equation (38) still holds, but there is no specific prediction for the term $\left\langle\omega_{j k} S^{j k}\right\rangle$. However it could be chosen to give the same results as equation (40), amongst other possibilities. On the other hand, both the vorticity and the angular velocitiy terms could satisfiy weak conditions similar to equation (38). Thus in the absence of any further information, one could assume that on the average, there are no correlations between the GR vorticity or the spin angular velocity with the spin. Then the average value of the effective spherically symmetric energy momentum becomes

$$
\left\langle T_{R}^{i j}\right\rangle_{I}=\left[\rho(1+\epsilon+p / \rho)-\frac{5}{6} \kappa S^{2}\right] u^{i} u^{j}+\left[p-\frac{7}{12} \kappa S^{2}\right] g^{i j}
$$

found in an earlier work [31].

\section{Conclusions}

We have seen through the investigation of self-consistent spinning fluids in a cosmological setting that the most active arena for spinning fluids is in a Riemann-Cartan spactime. Spinning fluids within the general relativistic context are very limited in the types of cosmologies that seem to exist [8]. In fact for certain assumptions on the cosmological model, the restrictive relation between the spin angular velocity and the spin given in equation (20) must be satisfied everywhere (not just on the average). Including the assumption of the vorticity-spin average given by equation (38) yields the effective energymomentum tensor of a perfect fluid given by equation (30). When the spinning fluids reside in a EC, a larger class of spacetimes are allowed. The less restrictive relationships given in equation (30) between the spin density and the spin angular velocity, due to the addition of spin-squared terms in the field equation, further enriches the character of the types of cosmologies that can exist. This leads to very general relationships amongst the spin density and the spin angular velocity, such as shown by equations (31) and (32) for arbitrary shear and expansion. If we include the vorticity assumption given by equation (38), then we obtain the effective energy-momentum tensor given by equation (40). It is in the $\mathrm{EC}$ case that one begins to see the importance of the consistency relations. Without using the consistency relations of the self-consistent theories, further assmptions on the averages of the spin angular velocity-spin terms must be made. In such cases, the average value of the improved energy-momentum tensor can be very different, as seen in equation (41), from the results for the classical Weyssenhoff fluid given in equation (40). However by including the consistency condition in the analysis, we find that the classical Weyssenhoff fluid is just one of several different possible spherically symmetric cosmologies with spin density in EC. This does not happen for spinning fluids in GR. 


\section{Addendum}

Special recognition is given to the referee who noticed that the angular velocity equation (5), which occurs in the consistency condition equation (27), is not as arbitrary as one might at first guess. Taking the traces of equations (5) and (32) and using the defintion of spin, he derives the relation $2 a^{1 t} \dot{a}_{i}{ }_{i}=\kappa \rho k_{0}$ between the tetrad spin angular velocity and the spin density in the fluid frame which must be satisfied in order to have a solution in the self consistent model used in this work. This observation solves a long time misconception on the arbitrariness of the angular velocity tensor in the self-consistent theories with extended thermodynamics.

An alternative approach to this problem used by the authors (and perhaps others) is to assume a metric form which can be as arbitrary as necessary. Consistent with this, a set of tetrads can be generated which then gives a dynamical basis for the spin angular velocity. This technique is very general within the limits of the metric and the spin density subsumed by the tetrads (see, for example, $[32,33]$ ).

\section{References}

[1] Ray J R 1972 J. Math. Phys. 131451

[2] Ray J R and Smalley L L 1982 Phys. Rev. D 262615

[3] Smalley L L 1993 Class. Quantum Grav. 101179

[4] Ray J R and Smalley L L 1982 Phys. Rev. Lett. 49 1059; 198350 626E; 1982 Phys. Rev. D 262619

[5] Amorim R 1984 Phys. Lett. 40A 259; 1985 Phys. Rev. D 313099

[6] de Ritis R, Lovorgna M, Platonia G and Stomaiola C 1985 Phys. Rev. D 311854

[7] Ray J R and Smalley L L 1986 Phys. Rev. D 343268

[8] Smalley L L and Krisch J P 1991 Class. Quantum Grav. 8 1889; 1992 J. Math. Phys. 331073

[9] de Ritis R, Lavorgna M, Platania G and Stornajolo C 1983 Phys. Rev. D28 713

[10] Obukhov Yu N and Korotky V A 1987 Class. Quantum Grav. 41633

[11] Obukhov Yu N and Piskareva O B 1989 Class. Quantum Grav. 6 L15

[12] Kopczynski W 1986 Phys. Rev. D34 352; 1990 Ann. Phys. 203308

[13] Obukhov Yu $N$ and Tresguerres R 1993 Hyperfluid-a model of classical matter with hypermomentum Preprint University of Cologne

[14] Kibble T W B 1961 J. Math Phys. 2212

Sciama D W 1962 Recent developments in General Relativity (Festschrift für Infeld) (Oxford: Pergamon) p 415

[15] Kopczynski W 1972 Phys. Leth. 39A 219

[16] Cartan E 1922 C. R. Acad. Sci. Paris 174593

[17] Nurgaliev IS and Ponomariev W N 1983 Phys. Lett. 130B 378

[18] Weyssenhoff J and Raabe A 1947 Acta Phys. Pol. 97

[19] Gasperini M 1986 Phys. Rev. Lett. 562873

[20] Martin M A P, Vasconcellos-Vaidya E P and Som M M 1991 Class. Quantum Grav. 82225

[21] Ray J R, Smalley L L and Krisch J P 1987 Phys. Rev. D 353261

[22] Smalley L L 1985 Phys. Rev. D 323124

[23] Krisch J P and Smalley L L 1988 J. Math. Phys. 291640

[24] Krisch J P and Smalley L L 1990 Class. Quantum Grav. 7481

[25] Misner C W, Thorne K S, and Wheeler J A 1973 Gravitation (San Francisco, CA: Freeman) section 6.5

[26] Smalley L L and Ray I R 1988 Phys. Leth. 134A 87

[27] Ehlers J and Kundt W 1962 Gravitation: an Introduction to Current Research ed L Witten (New York; Wiley) p 57

[28] Schouten J A 1954 Ricci Calculus (Berlin: Springer)

[29] Fennelly A J, Krisch J P, Ray J R and Smalley L L 1991 J. Math. Phys. 32485

[30] Smalley $L L$ and Krisch J P On spherically symmetric cosmologies in Riemann-Cartan spacetime with spin density Report 8.7.92 University of Alabama in Huntsville

[31] Heht F W, von der Heyde P and Kerlick G D 1974 Phys. Rev. D 101066

[32] Krisch J P and Smalley L L 1990 Class. Quantum Grav. 7 481; 1988 J. Math. Phys. 291640

[33] Smalley L L 1985 Phys. Rev. D 323124 